\title{
Orlistat: una nueva herramienta farmacológica en el tratamiento de la obesidad
}

Randomised Placebo-controlled trial of orlistat for weight loss and prevention of weight regain in obese patients. Sjostrom L, Rissanen A, Andersen T, et al. Lancet 1998; 352:167-73.

\section{Objetivo}

Evaluar la eficacia y tolerancia del orlistat en el tratamiento de la obesidad. Secundariamente se evaluaron los efectos sobre los factores de riesgo cardiovascular (FRC).

\section{Diseño}

Ensayo clínico randomizado controlado doble ciego, con grupos paralelos. Tiempo de seguimiento: 2 años.

\section{Lugar}

15 centros de atención ambulatoria.

\section{Pacientes}

Se invitaron a participar a 743 pacientes voluntarios $>18$ años con índice de masa corporal (IMC) entre 28 y 47 . Se excluyeron pacientes con hipertensión no controlada, diabetes tratada con fármacos, pérdida de peso mayor a $4 \mathrm{~kg}$. en los últimos 3 meses, cirugía para reducción de peso, bridas intestinales, bulimia, abuso de drogas o alcohol, y mujeres en edad fértil sin método anticonceptivo.

\section{Intervención}

Se incluyeron 688 pacientes bien predispuestos (que cumplieron satisfactoriamente un período de reclutamiento recibiendo sólo placebo y dieta hipocalórica) fueron randomizados a orlistat $120 \mathrm{mg}$ o placebo 3 veces por día durante 1 año; asociado a una dieta ligeramente hipocalórica (600 kcal/día por debajo del gasto calórico calculado para cada paciente).

En el segundo año los pacientes con mejor adherencia al tratamiento (>75\%) fueron reasignados a orlistat o placebo, asociado en este segundo año a una dieta eucalórica. Así, al cabo de los 2 años quedaron 4 grupos para el análisis: orlistat-orlistat, orlistat-placebo, placeboplacebo, placebo-orlistat (1er y 2 do año respectivamente).

\section{Medición del resultado principal}

Reducción de peso en el primer año y mantenimiento de peso en el segundo. El análisis fue realizado por intención de tratar*.
Modificación de FRC y efectos adversos. Se realizó periódicamente dosaje de colesterol total, colesterol LDL y HDL, triglicéridos, insulina y glucemia, además de dosaje de vitaminas.

\section{Resultados Principales}

El 79\% de los participantes completaron el 1er año. Los dos años fueron completados por el $63 \%$ de los randomizados.

Reducción y mantenimiento de peso: Al final del primer año, la reducción de peso promedio (desde el reclutamiento) fue de $10.3 \mathrm{~kg}$. (el $10.1 \%$ del peso) para el grupo orlistat y de $6.1 \mathrm{~kg}$. (6.1\% del peso) para el grupo placebo $(p<0.001)$. Perdieron mas del $20 \%$ del peso basal un $9.3 \%$ de los pacientes del grupo orlistat vs. $2.1 \%$ del placebo ( $p<0.001)$; y del 10 al $20 \%$ del peso un $29.5 \%$ y un $15.6 \%$ respectivamente $(p<0.001)$.

En el segundo año todos lo grupos (excepto placebo-orlistat) recuperaron peso. El grupo placebo-orlistat tuvo una reducción de peso de $3.6 \mathrm{~kg}$. (p 0.001). En los pacientes orlistat-orlistat la reganancia de peso fue menor que en el grupo orlistat-placebo, con una diferencia de $2.4 \mathrm{~kg}(\mathrm{p}<0.001)$. FRC: La disminución de peso observada en el grupo placebo durante el reclutamiento, se acompañó de disminución del colesterol, LDL, HDL, glucemia y tensión arterial. Durante el estudio, estos parámetros retornaron a valores previos en el grupo placebo (colesteroly LDL) y no en el orlistat. El descenso del colesterol fue independiente del descenso de peso.

Efectos adversos: fueron mayores en el grupo orlistat. Los gastrointestinales fueron los más relevantes y de mayor frecuencia en el primer año. Comparando grupo orlistat vs. placebo, la esteatorrea se presentó en el 31 y $5 \%$, diarrea 13 y 10\%, urgencia fecal 10 y 3\%, flatulencia 7 y $3 \%$, incontinencia fecal 7 y $0 \%$ respectivamente. Debieron recibir suplementos de vitaminas liposolubles, 16 pacientes del grupo orlistat y 4 del grupo placebo.

\section{Conclusiones}

El orlistat fue eficaz y seguro en el descenso y mantenimiento de peso, asociado a una dieta adecuada por un lapso de 2 años en pacientes muy adherentes. Estos efectos se asociaron también a un mejor perfil de los FRC.

\section{COMENTARIO}

La obesidad es una enfermedad multicausal y compleja, aun no totalmente conocida. Un inhibidor de la lipasa intestinal como el orlistat no es capaz de modificar la etiopatogenia de la enfermedad, por lo que no debe sustituir a los otros recursos sino, complementarlos. Actualmente las únicas armas terapéuticas útiles para el descenso de peso son un adecuado plan alimentario, la actividad física sistemática y el abordaje grupal interdisciplinario. Los predictores de éxito en el mantenimiento del peso a largo plazo son la incorporación de actividad física como hábito y la capacidad de autorregistro que se enseña en los grupos terapéuticos. Aun así, el éxito es infrecuente, si nos referimos a la población que habitualmente se presenta con esta patología. Los pacientes que se seleccionaron para este estudio constituyen un subgrupo de todos los obesos, muy motivados para bajar de peso y cumplir con las indicaciones médicas. Esto queda reflejado

*Ver Glosario

\section{Dra. Verónica Schoj}

Unidad de Medicina Familiar. Hospital Italiano Buenos Aires. en la elevadísima tasa de adherencia a la dieta restrictiva al año y a los dos años, que no concuerda con los reportes generales de la literatura (porcentaje de abandono entre el 70 y $80 \%$ al año). Esto condiciona la validez externa* del estudio.

Será necesario esperar nuevos ensayos que evalúen su eficacia y seguridad a más largo plazo y en pacientes con características de adherencia más parecidas a las de la práctica cotidiana.

El orlistat ha mostrado ser eficaz en este ensayo, pero su indicación indiscriminada aún no ha demostrado estar libre de riesgos. Podría, en cambio, ser un elemento coadyuvante en un grupo seleccionado de pacientes adherentes al plan alimentario y a la actividad física, con un adecuado entendimiento de la enfermedad y cuyo objetivo primario sea la reducción de la morbimortalidad y no el alcance de los estándares estéticos actuales. 